\title{
The location-, word-, and arrow-based Simon effects: An ex-Gaussian analysis
}

\author{
Chunming Luo ${ }^{1} \cdot$ Robert W. Proctor ${ }^{2}$
}

Published online: 20 November 2017

(C) Psychonomic Society, Inc. 2017

\begin{abstract}
Task-irrelevant spatial information, conveyed by stimulus location, location word, or arrow direction, can influence the response to task-relevant attributes, generating the location-, word-, and arrow-based Simon effects. We examined whether different mechanisms are involved in the generation of these Simon effects by fitting a mathematical ex-Gaussian function to empirical response time (RT) distributions. Specifically, we tested whether which ex-Gaussian parameters $(\mu, \sigma$, and $\tau$ ) show Simon effects and whether the location-, word, and arrow-based effects are on different parameters. Results show that the locationbased Simon effect occurred on mean RT and $\mu$ but not on $\tau$, and a reverse Simon effect occurred on $\sigma$. In contrast, a positive word-based Simon effect was obtained on all these measures (including $\sigma$ ), and a positive arrow-based Simon effect was evident on mean RT, $\sigma$, and $\tau$ but not $\mu$. The arrow-based Simon effect was not different from the wordbased Simon effect on $\tau$ or $\sigma$ but was on $\mu$ and mean RT. These distinct results on mean RT and ex-Gaussian parameters provide evidence that spatial information conveyed by the various location modes are different in the timecourse of activation.
\end{abstract}

Keywords Location modes $\cdot$ Simon effect $\cdot$ Ex-Gaussian

Chunming Luo

luocm@psych.ac.cn

1 CAS Key Laboratory of Behavioral Science, Institute of Psychology, 16 Lincui Road, Chaoyang District, Beijing 100101, China

2 Department of Psychological Sciences, Purdue University, West Lafayette, IN, USA

\section{Introduction}

When people respond to an object or its features, the location occupied by the object can influence the response, which is usually addressed in the Simon task, named for J. R. Simon (1990). In the typical visual version of this task with horizontal stimulus-response (S-R) arrangement, left and right stimulus locations are task-irrelevant and non-spatial attributes (e.g., colors or shapes) convey the task-relevant information, to which left and right keypresses are mapped arbitrarily. Response performance is better when the non-spatial attributes and response positions correspond than when they do not, a finding that is called the spatial or location-based Simon effect (see reviews of Lu \& Proctor, 1995; Proctor, Miles, \& Baroni, 2011; Simon, 1990; Umiltà \& Nicoletti, 1990). The Simon effect also occurs with stimuli whose task-irrelevant spatial information is conveyed not by their physical locations but by the meanings of centrallypresented location words or arrows, resulting in wordand arrow-based Simon effects (Khalid \& Ansorge, 2013; Lu \& Proctor, 2001; Pellicano, Lugli, Baroni, \& Nicoletti, 2009; Proctor, Marble, \& Vu, 2000).

The location-based Simon effect has been attributed to responses primed by automatic processing of task-irrelevant stimulus location (direct route), which interferes with responses activated by processing of a task-relevant attribute in accordance with the instructed S-R mapping (indirect route; De Jong, Liang, \& Lauber, 1994; Hommel, 1997; Kornblum, Hasbroucq, \& Osman, 1990; Ulrich, Schröter, Leuthold, \& Birngruber, 2015; Zhang, Zhang, \& Kornblum, 1999; Zorzi \& Umiltà, 1995), which is named the dual-route model. Similarly, given that the location words and arrow directions can be processed automatically (Hommel, Pratt, Colzato, \& Godijn, 2001; Lu \& Proctor, 1995), word- and arrow-based Simon effects are ascribed to the automatically 
processed location word and arrow direction interfering with the incongruent response indicated by their ink colors (Lu \& Proctor, 2001; Luo \& Proctor, 2016; Proctor et al., 2000; Proctor, Yamaguchi, Zhang, \& Vu, 2009).

\section{Response-time distribution and chronometric analyses}

Initial studies of the Simon effect and related correspondence effects focused on differences in mean response time (RT) that provide estimates of the central tendency for each condition (see Lu \& Proctor, 1995), which are still the focal point of many studies. One problem for analyses limited to mean RT is that it is difficult to specify thoroughly how the experimental manipulation influences RT. On the one hand, the increase of mean RT resulting from non-correspondence may be due to a shift of the RT distribution (see location-based Simon in Fig. 1), an increase in the skew of the RT distribution in one condition compared to the other condition (Tse, Balota, Yap, Duchek, \& McCabe, 2010), or both (see word- and arrowbased Simon effects in Fig. 1). On the other hand, RTs may be affected by manipulations that have no obvious effect on mean RT but on the skew of the RT distribution (Spieler, Balota, \& Faust, 2000). Therefore, beginning with De Jong et al. (1994), reporting analyses of the Simon effect across the RT distributions (delta plots) has become common (e.g., Davranche \& McMorris, 2009; Kubo-Kawai \& Kawai, 2010). In studies using a Vincentizing procedure (Ratcliff, 1979), a group RT distribution is obtained by partitioning each participant's RTs on the corresponding and noncorresponding trials into percentile bins (e.g., 10), ranging from shortest to longest, and the Simon effect (the difference) is calculated for each bin. Most findings are that the location-based Simon effect is largest for fast responses but decreases, and even may reverse, as responses slow when the imperative stimulus is displayed in a left or right location (Ansorge \& Wühr, 2004; Ellinghaus, Karlbauer, Bausenhart, \& Ulrich, 2017; Pratte, Rouder, Morey, \& Feng, 2010; Servant, Montagnini, \& Burle, 2014; Ulrich et al., 2015). In contrast, the word- and arrow-based Simon effects increase as RTs increase (Miles \& Proctor, 2012; Pellicano et al., 2009; Proctor et al., 2009).
Likewise, in a range of other tasks and conditions, there is growing interest in fitting a mathematical function to empirical RT distributions to quantify their characteristics in order to allow measurement of more than central tendency (Heathcote, Popiel, \& Mewhort, 1991; Hockley, 1984; Ratcliff, 1978, 1979; Spieler, Balota, \& Faust, 1996; Spieler et al., 2000; White, Risko, \& Besner, 2016; Yap, Balota, Tse, \& Besner, 2008). The convolution of a Gaussian and an exponential distribution, called the ex-Gaussian distribution, has a good fit to empirical RT distributions in a range of tasks (Heathcote et al., 1991; Hockley, 1984; Hohle, 1965; Spieler et al., 1996), and the stability of the parameter estimates has been most fully demonstrated in Ratcliff s $(1978,1979)$ pioneering work in testing an explicit model of memory retrieval, and in other studies with the Stroop task paradigm (Heathcote et al., 1991; Spieler et al., 1996; Spieler et al., 2000; Steinhauser \& Hübner, 2009).

Fitting the ex-Gaussian distribution to an empirical RT distribution results in estimates of three parameters $(\mu, \sigma$, and $\tau)$. The parameter $M$ reflects the mean of the Gaussian component of the distribution. A change in $\mu$ reflects a shift of the RT distribution between different conditions, and it indicates that a variable has an early acting influence on the majority of RTs. $\sigma$ reflects the standard deviation associated with the Gaussian component, and a change in $\sigma$ reflects the differences of standard deviation of the RT distributions between different conditions. $\tau$ reflects the mean and standard deviation of the exponential component. A change in $\tau$ reflects the change in the tail of the RT distribution between different conditions, and it indicates that a variable has a late-acting influence on some trials. Importantly, the mean of the ex-Gaussian distribution equals $\mu+\tau$, and its variance equals $\mu^{2}+\tau^{2}$.

The ex-Gaussian analysis offers another way of characterizing the influence of factors on RT distributions, and these ex-Gaussian parameters provide more information about changes in the RT distribution than are reflected in analyses of mean RTs and delta plot functions (Andrews \& Heathcote, 2001; Balota \& Spieler, 1999; Heathcote, Popiel, \& Mewhort, 1991; Spieler et al., 1996; White et al., 2016). For example, ex-Gaussian distribution analysis can decompose effects of response- and task-related conflicts in an
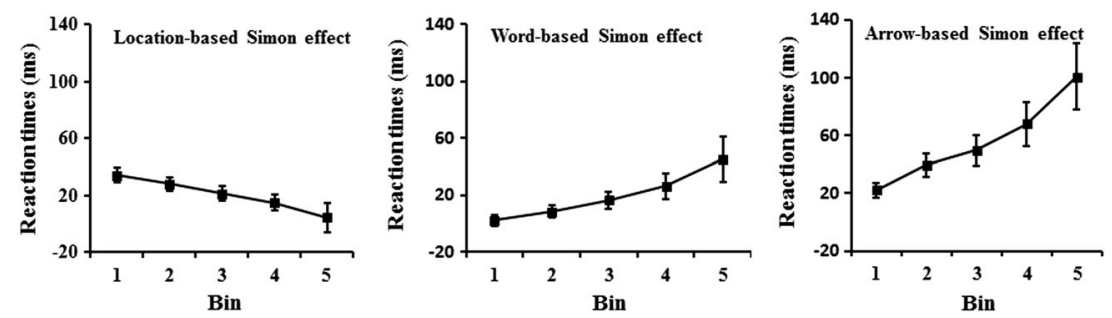

Fig. 1 Mean response times and standard errors for each of the bins for the location-based Simon effect in Experiment 1, the word-based Simon effect in Experiment 2, and the arrow-based Simon effect in Experiment 3 
across-task associative priming paradigm that are not distinguishable in analyses of mean RTs (Moutsopoulou \& Waszak, 2012). This decomposition is possible because conflict related to stimulus-response associations retrieved after a switch of tasks is reflected in the Gaussian component ( $\mu$ and $\sigma)$, whereas conflict related to the retrieval of stimulus-task associations is reflected in the exponential component $(\tau)$. Moreover, ex-Gaussian distribution analysis allows one to see whether some experimental effect on mean RT arises from an early shift of the RT distribution (a change in $\mu$ ), an increase of late responses in the tail of the RT distribution (a change in $\tau$ ), or both (Tse et al., 2010). For example, an exGaussian analysis revealed that a semantically-based Stroop effect (effect of incongruent color-associated word on responding to color) was restricted to the arithmetic mean and $\mu$ but not $\tau$ (White et al., 2016), suggesting that the semantically-based Stroop effect on mean RT arises from the early faster responses. By contrast, the typical colorword Stroop effect is obtained on all three ex-Gaussian parameters (Spieler et al., 1996, 2000), providing evidence that the effect occurs early and lasts for a long time. Moreover, we can obtain $\sigma$ for each condition and, by calculating their differences, can determine whether the delta plot is positive or negative (Zhang \& Kornblum, 1997).

\section{Mode-specific or shared mechanism}

There is agreement that spatial locations activate visual-spatial codes, and location words are symbolic and activate semanticspatial codes (i.e., codes of spatial meaning; Hommel et al., 2001; Notebaert, De Moor, Gevers, \& Hartsuiker, 2007). Arrows are usually regarded as symbolic (e.g., Freitas, Bahar, Yang, \& Banai, 2007; Tipples, 2002), although they also have visual-spatial properties (e.g., for a left-pointing arrow, the arrowhead is located to the left of the shaft). Therefore, arrows may activate visual- and semantic-spatial codes. Although the spatial codes activated by various spatial modes are different, they may indicate the same concepts (e.g., left or right) and have shared representations, as evidenced in some studies (e.g., De Houwer, Beckers, Vandorpe, \& Custers, 2005; Notebaert et al., 2007; Proctor et al., 2009). Both location words and arrows yield S-R mapping effects when the location information conveyed by them is relevant (Wang \& Proctor, 1996) and Simon effects when it is irrelevant (Proctor \& Vu, 2002). Moreover, the locationbased Simon effect is eliminated when location-based Simon task trials are intermixed with ones in which participants respond to the words left and right with incompatibly mapped keypresses (Notebaert et al., 2007; Proctor et al., 2000; Vu, Ngo, Minakata, \& Proctor, 2010).

The different spatial codes, generated by various location modes sharing spatial representations, triggered an issue of whether the spatial information conveyed by these various stimulus modes influences responses in a similar way or with identical processes. Some findings suggest that different location modes affect responses with modespecific mechanisms, as the Simon effects based on them seem to have different temporal dynamics. For example, the location-based Simon effect decreases as RTs increase across the RT distribution for a left- or right-displayed imperative stimulus, whereas the word- and arrow-based Simon effects increase as RTs increase (Miles \& Proctor, 2012; Pellicano et al., 2009; Proctor et al., 2009). Wascher and colleagues (Wascher, Schatz, Kuder, \& Verleger, 2001; Wiegand \& Wascher, 2005, 2007) suggested that increasing and decreasing delta plots of Simon effects obtained in various tasks are the result of two distinct mechanisms. One is responsible for a "visuomotor Simon effect" underlying the location-based Simon task, and the other for a "cognitive Simon effect" occurring in other forms of the Simon task, which is the product of high-level cognitive interference. The visuomotor Simon effect arises from the stimulus location automatically activating the corresponding response via the visuomotor pathway (Wascher et al., 2001; Wiegand \& Wascher, 2005, 2007), and the activation quickly fades out over time, resulting in a decreasing delta plot. The cognitive Simon effect increases over time as the task-irrelevant activation is slower and does not decay, leading to a positive delta plot.

In contrast to the explanation of two different mechanisms, some studies have proposed that even though the slopes of the delta plots are different in variants of the task, their underlying mechanism is the same. For example, Töbel, Hübner, and Stürmer (2014) found that the correspondence of the previous trial had a similar effect on the delta plots of horizontal and vertical visual Simon tasks. In both tasks, the slopes of the slowest quantiles of the delta plot decreased more following non-corresponding trials and the accuracy in the fastest quantiles was higher after incompatible trials than it was after compatible trials.

Studies of cognitive computational models also suggest that the location-based Simon effect (with a decreasing delta plot) and flanker effect, and Stroop effect for which the delta plot is increasing) can be fit by the same models (Servant, Montagnini, \& Burle, 2014; Ulrich et al., 2015). According to the diffusion model for conflict tasks (Ulrich et al., 2015), the shape of delta functions largely varies with the relative speeds of the automatic activation and the controlled processes. Automatic activation of stimulus location developing quickly makes the negative slope of the delta functions of location-based Simon effect, whereas color word and arrow activation developing slowly make the positive slope of the delta functions of the flanker and color-word Stroop effects. These studies argue for a single underlying mechanism as the most parsimonious explanation to the time course of the Simon effect. 


\section{Present study}

In the current study, we examined whether these various Simon effects occur on each of the ex-Gaussian parameters, and whether there are differences between them. To our knowledge, no studies have used the ex-Gaussian analysis to explore the Simon effect, particularly for the arrow- and wordbased Simon effects. Given that ex-Gaussian distribution analysis can isolate the differential effects of task and response conflict in priming paradigms (Moutsopoulou \& Waszak, 2012) and of color-word and semantic Stroop effects (Spieler et al., 1996, 2000; White et al., 2016), we expected that it could distinguish different Simon effects.

Specifically, in Experiment 1, the location-based Simon task with horizontal S-R arrangement was used. Given that the location-based Simon effect is largest for fast responses but decreases for slower responses across the RT distribution (Ansorge \& Wühr, 2004; Pratte et al., 2010), the Simon effect on mean RT may mainly arise from a shift of the RT distribution ( $\mu$ in the corresponding condition is smaller than that in the noncorresponding condition) and little from the tail of the RT distribution. Moreover, previous studies have demonstrated that effects of response conflict were mainly observed on $\mu$ and $\sigma$ but not $\tau$ (Spieler et al., 2000; Steinhauser \& Hübner, 2009; White et al., 2016). Thus, the location-based Simon effect would be expected to occur on $\mu$ but not $\tau$, given that the effect is mainly associated with response selection $(\mathrm{Lu} \&$ Proctor, 1995). These expected results would help to test the diffusion model for conflict tasks (Ulrich et al., 2015), which assumes that automatic activation of stimulus location develops quickly, producing a location-based Simon effect in fast responses but not slow responses.

In Experiments 2 and 3 we examined separately the wordand arrow-based Simon effects on ex-Gaussian parameters. Given that the word- and arrow-based Simon effects increase as RTs increase (Miles \& Proctor, 2012; Pellicano et al., 2009; Proctor et al., 2009), they may arise from a shift of the RT distribution and from the tail of the RT distribution in the incompatible condition, compared to that in the compatible condition. Thus, we predicted that these Simon effects would occur on $\mu$ and $\tau$. These expected results would also help support the diffusion model for conflict tasks (Ulrich et al., 2015), which assumes that automatic activation of words and arrows develops across time, resulting in their associated Simon effects occurring in both the fast and slow responses.

\section{Experiment 1: Location-based Simon effect}

\section{Method}

Participants Twenty right-handed participants (eight males), aged 18-24 years, took part in Experiment 1. All participants in this experiment and the others were native speakers of Chinese (Putonghua), had normal or correctedto-normal vision, and were naïve as to the purpose of the experiment.

Apparatus, stimuli, procedure, design Stimuli were presented on a super VGA high-resolution color monitor with grey background. A personal computer, running E-Prime 1.1 software, controlled the presentation of stimuli, timing operations, and data collection. Participants placed their heads on a chinrest and viewed the monitor from a distance of $58 \mathrm{~cm}$ in a dimly lit room. The stimuli were red and a green squares $\left(0.85^{\circ} \times 0.85^{\circ}\right)$, whose centers were $4.0^{\circ}$ to the left or right of the center of screen.

Each participant performed eight practice trials followed by 128 test trials (64 compatible and 64 incompatible, randomly intermixed). Each trial began with onset of a white central fixation cross $\left(0.4^{\circ} \times 0.4^{\circ}\right)$. After $1 \mathrm{~s}$, a square in red or green appeared for $150 \mathrm{~ms}$. After that, the gray screen became blank, during which the trial terminated if the participant responded or after $1.5 \mathrm{~s}$ if no response had been made. After the response, a 1-s inter-trial interval occurred, during which the screen remained blank. Responses were made by pressing a left key (V) for the red ink color or right key (M) for the green ink color on the computer keyboard with the left or right index finger. The mapping of colors to left or right responses was counterbalanced across participants. The response keys and computer screen were aligned such that the fixation point and the midway point between the two response keys were on the participant's sagittal midline. Participants were instructed to maintain fixation and to respond to the targets as quickly and accurately as possible.

Data analysis The practice trials were excluded from the RT and percent error (PE) analyses in Experiments 1-3. In Experiment 1, the following test trials were excluded from the RT (including Vincentile analysis and ex-Gaussian) analyses: those with (a) incorrect response to the target (3.8\%), (b) RTs longer than $1,500 \mathrm{~ms}$ or shorter than $150 \mathrm{~ms}(0.0 \%)$, and (c) outside of three standard deviations of the mean for each condition per participant (1.3 $\%)$. Mean correct RTs and PEs in Experiments 1-3 are then presented in Table 1.

Secondly, using the Vincentizing procedure (Ratcliff, 1979), RTs were rank ordered from shortest to longest in each condition for each participant in Experiments 13 , divided into five equally sized bins and averaged in each bin. The Simon effects for each spatial mode were then calculated for each bin in Experiments 1-3, as shown in Fig. 2. An analysis of variance (ANOVA) was performed on RT, with bin and location-based Simon (compatible, incompatible) as within-subject variables. Considering the way in which the RT data were 
Table 1. Mean percentage of error (PE), mean response time (RT, in $\mathrm{ms}$ ), $\mu, \sigma, \tau$, and standard deviation (in parentheses) for the compatible and incompatible conditions in the location-based Simon (Experiment 1), word-based Simon (Experiment 2), and arrow-based Simon (Experiment 3) tasks

\begin{tabular}{|c|c|c|c|c|c|c|}
\hline & & $\mathrm{PE}$ & $\mathrm{RT}$ & $\mu$ & $\sigma$ & $\tau$ \\
\hline \multirow[t]{2}{*}{ Experiment 1} & Compatible & $3.3(.03)$ & $491(61)$ & $445(54)$ & 61(19) & $47(15)$ \\
\hline & Incompatible & $4.4(.04)$ & $512(65)$ & $468(54)$ & $49(16)$ & $44(15)$ \\
\hline \multirow[t]{2}{*}{ Experiment 2} & Compatible & $1.5(0.03)$ & $461(79)$ & $403(71)$ & $65(19)$ & $58(32)$ \\
\hline & Incompatible & $2.5(0.04)$ & $485(96)$ & $415(80)$ & $76(33)$ & $71(35)$ \\
\hline \multirow[t]{2}{*}{ Experiment 3} & Compatible & $2.2(0.06)$ & $414(82)$ & $376(64)$ & $44(20)$ & $38(21)$ \\
\hline & Incompatible & $7.4(0.07)$ & $470(124)$ & $414(92)$ & $64(37)$ & $57(44)$ \\
\hline
\end{tabular}

grouped, the bin main effect was significant in all analyses, so it was not reported and discussed here or in the following experiments.

Lastly, the same correct RT data were fit to the exGaussian function for each participant in each condition by using the timefit function in the retimes package (Massidda, 2013). The timefit function uses maximum likelihood estimation to generate the three parameters $(\mu, \sigma, \tau)$. Separate repeated-measures ANOVAs were performed on these parameters, with word-based Simon and location-based Simon as within-subject variables.
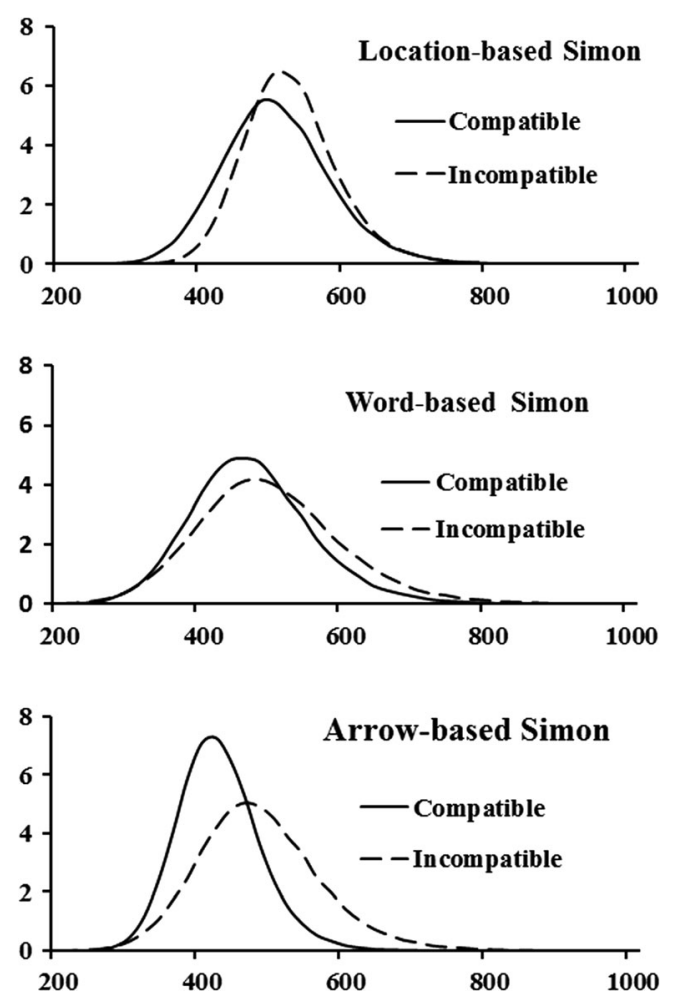

Fig. 2 The probability density functions separately for compatible and incompatible conditions in the location-based Simon task in Experiment 1 (top panel) and in the word-based Simon task in Experiment 2 (middle panel), and in the arrow-based Simon task in Experiment 3 (bottom panel)

\section{Results}

PE. There was a Simon effect of $1.3 \%$, with fewer response errors in the compatible $(3.1 \%)$ than in the incompatible condition $(4.4 \%)$, but the difference was not statistically significant, $t(19)=1.07, p=.297$.

RT. Vincentile analysis. The main effect of location-based Simon was significant, $F(1,19)=17.43, p=.001, M S E=$ $1,218, \eta_{p}^{2}=.479$, reflecting a Simon effect of $21 \mathrm{~ms}$. The interaction between bin and location-based Simon was significant, $F(4,76)=6.00, p<.001, M S E=223, \eta_{p}{ }^{2}=.240$. Further analysis showed that the Simon effect was not significant for bin 5, $p=.672$, but was for the other bins, $p s<.017$. Trend analysis showed that the Simon effect decreased linearly from bin 1 to bin 5 (see Fig. 2), $F(1,19)=7.59, p=.013$, $M S E=1,389, \eta_{p}{ }^{2}=.286$.

Ex-Gaussian analysis. The location-based Simon effect was significant on $\mu, t(19)=4.53, p<.001$, with a $23 \mathrm{~ms}$ Simon effect, and $\sigma, t(19)=-2.41, p=.026$, with a $-12 \mathrm{~ms}$ Simon effect, but not on $\tau, t(19)=1.04, p=.311$.

\section{Discussion}

The location-based Simon effect was obtained on mean RT, and the effect decreased as RT increased in the Vincentile analyses, replicating findings obtained in previous studies (Ansorge \& Wühr, 2004; Pratte et al., 2010; Proctor et al., 2009; Ellinghaus et al., 2017). The location-based Simon effect of $21 \mathrm{~ms}$ was similar to Ellinghaus et al.'s Experiment $1(22 \mathrm{~ms})$ and Experiment 2 (24 ms), in which the location-based Simon effect on mean RT and delta functions were invariant against changes of stimulus duration (150 ms or until response emitted). Likewise, as predicted, we obtained the location-based Simon effects on $\mu$ and $\sigma$, but not on $\tau$, consistent with the findings that response conflict is mainly observed on $\mu$ and $\sigma$ but not on $\tau$ (Spieler et al., 1996; Steinhauser \& Hübner, 2009; White et al., 2016).

The location-based Simon effect occurred on $\mu$ but not on $\tau$, which showed that the effect on mean RT is because the RT 
distribution in the incompatible condition, compared to that in the compatible condition, shifts from left to right, as shown in Fig. 1. These results suggest that the location-based Simon effect mainly arises from the faster responses and not from the slower responses. Likewise, the effect on $\sigma$ was negative, showing that variance was larger in the compatible condition than in the incompatible condition, as shown in Fig. 1. The larger variance in the compatible condition results in a decreasing delta plot function, as shown in Fig. 2, compatible with the mathematical proof in Zhang and Kornblum (1997), which showed that the reduction in location-based Simon effect with an increase of RT occurs when the variance of the RT distribution for compatible trials is larger than that for incompatible trials.

\section{Experiment 2: Word-based Simon effect}

\section{Method}

Twenty new right-handed participants (eight males), aged 1926 years, took part in Experiment 2. The apparatus, stimuli, procedure, design and data analysis were identical to Experiment 1, except that the red and green squares were replaced by Chinese single-character words 左 (left) and 右 (right) $\left(0.85^{\circ} \times 0.85^{\circ}\right)$ of high frequency $(2,404$ and 1,856 in Modern Chinese Frequency Dictionary, 1985). They were written in red or green and presented on the center of the screen, and responses were made to their colors. Experiment 2 had a variable, word-based Simon (compatible, incompatible), with 64 observations per experimental condition.

\section{Results}

As in Experiment 1, test trials wherein participants responded incorrectly to the target $(2.1 \%)$ and RTs longer than $1,500 \mathrm{~ms}$ or less than $150 \mathrm{~ms}(0.4 \%)$ were excluded from the RT analyses, and test trial RTs that were not within three standard deviations of the mean $(2.2 \%)$ for each condition per participant were excluded from the RT analyses.

PE. The word-based Simon effect was significant, $t(19)=$ $3.12, p=.006$, with fewer errors in the compatible condition $(1.5 \%)$ than in the incompatible condition $(3.5 \%)$.

RT. Vincentile analysis. The word-based Simon effect was significant, $F(1,19)=9.78, p=.006, M S E=2,025, \eta_{p}{ }^{2}=$ .340 , which reflects a Simon effect of $24 \mathrm{~ms}$. Moreover, the interaction between bin and word-based Simon was significant, $F(4,76)=5.80, p<.001, M S E=488, \eta_{p}{ }^{2}=.234$. Further analysis showed that the location-based Simon effect was not significant for bin $1, t(19)=.64, p=.529$, approached significance for bin $2, t(19)=2.02, p=.057$, and was significant for bins 3 to $5, p s<.011$. Trend analysis revealed that the word- based Simon effect increased linearly from bin 1 to bin 5 , $F(1,19)=6.36, p=.021, M S E=3,368, \eta_{p}{ }^{2}=.251$ (see Fig. 2). In addition, a comparative analysis showed that the word-based Simon effect on mean RT was not different from the location-based Simon effect in Experiment 1, $t(38)=.06, p=.952$.

Ex-Gaussian analysis The word-based Simon effect was significant on $\mu, t(19)=2.26, p=.036$, on $\sigma, t(19)=2.24, p=$ .037 , and on $\tau, t(19)=2.13, p=.047$, indicating $12 \mathrm{~ms}, 11 \mathrm{~ms}$ and $13 \mathrm{~ms}$ Simon effects on these parameters, separately. An analysis comparing Experiments 1 and 2 showed that the word-based Simon effect was smaller than the locationbased Simon effect on $\mu, t(38)=2.10, p=.042$, but larger than it on $\sigma, t(38)=3.24, p=.002$, and on $\tau, t(38)=2.35, p=$ .024. In addition, the word-based Simon effect on $\sigma$ was not different from the absolute value of the location-based Simon effect, $t(38)=.14, p=.892$.

\section{Discussion}

The word-based Simon effect was obtained on mean RT, and the effect became larger as RT increased in the Vincentile analyses. This pattern replaces findings obtained for keypress responses in previous studies (Experiment 2 in Khalid \& Ansorge, 2013; Pellicano et al., 2009; Proctor et al., 2009, 2011), although left-right eye-movement responses have been shown to yield a decreasing delta plot (Khalid \& Ansorge, 2013).

As predicted, we obtained the word-based Simon effects on $\mu, \sigma$, and $\tau$. The word-based Simon effect on RT may arise from a shift of the RT distribution and from the tail of the RT distribution in the incompatible condition, compared to that in the compatible condition, suggesting that the word-based Simon effect occurred in both fast and slow responses. Compared to the location-based Simon effect in Experiment 1, the word-based Simon effect was not different from it on mean RT, but was smaller than it on $\mu$ and larger than it on $\tau$, suggesting that the word-based Simon effect has a greater contribution from the slow responses. Moreover, the wordbased Simon effect on $\sigma$ was positive, whereas the locationbased Simon effect was negative, but the absolute value was not different: The variances across the RT distributions were not different for the two tasks, but the direction of the effect was opposite.

\section{Experiment 3: Left- or right-pointing arrows}

\section{Method}

Twenty new right-handed subjects (nine males), aged 18-24 years, took part in Experiment 3. Apparatus, stimuli, 
procedure, design were identical to Experiment 2, except that the location words were replaced with green or red left- or right-pointing arrows $\left(\rightarrow\right.$ and $\rightarrow, 0.85^{\circ} \times 0.85^{\circ}$, with $0.51^{\circ} \times$ $0.85^{\circ}$ for arrow head and $0.34^{\circ} \times 0.15^{\circ}$ for arrow bar), and responses were made to their colors.

\section{Results}

Test trials with incorrect response to the target (3.2\%) and RTs longer than $1,500 \mathrm{~ms}$ or less than $150 \mathrm{~ms}(1.6 \%)$, and test trials RTs were not within three standard deviation of mean $(2.1 \%)$ for each condition per participant were excluded from the RT analyses.

PE. The main effect of arrow-based Simon was significant, $t(19)=5.25, p<.001$, indicating a Simon effect of $5.2 \%$, with fewer response errors in the compatible $(2.2 \%)$ than in the incompatible condition ( $7.4 \%)$.

RT. Vincentile analysis. The arrow-based Simon effect was significant, $F(1,19)=23.40, p<.001, M S E=6,699, \eta_{p}{ }^{2}=$ .552 , reflecting a Simon effect of $56 \mathrm{~ms}$. Moreover, the interaction between bin and arrow-based Simon was significant, $F(4,76)=11.91, p<.001, M S E=760, \eta_{p}^{2}=.385$. The Simon effect was significant for each bin, $p s<.001$. Trend analysis showed that the arrow-based Simon effect increased linearly from bin 1 to bin $5, F(1,19)=13.27, p=.002, M S E=$ $5,049, \eta_{p}^{2}=.419$ (see Fig. 2). In addition, a comparison analysis showed that the arrow-based Simon effect on mean RT was larger than both the location-based Simon effect in Experiment $1, t(38)=2.85, p=.007$, and the word-based Simon effect in Experiment 2, $t(38)=2.77, p=.009$.

Ex-Gaussian analysis. The arrow-based Simon effect was significant on $\mu, t(19)=4.30, p<.001$, on $\sigma, t(19)=3.40, p=$ .003 , and on $\tau, t(19)=2.84, p=.010$, indicating $37 \mathrm{~ms}, 20 \mathrm{~ms}$, and $18 \mathrm{~ms}$ Simon effects on these parameters, separately. Compared to the location-based Simon effect in Experiment 1, the arrow-based Simon effect was larger on $\sigma, t(38)=4.16$, $p<.001$, and on $\tau, t(38)=3.00, p=.005$, but not on $\mu, t(38)=$ $1.34, p=.188$. In addition, there also was no difference in absolute value of $\sigma, t(38)=1.21, p=.234$, whereas the direction of the effect is reverse. Moreover, the arrow-based Simon effect was larger than the word-based Simon effect in Experiment 2 on $\mu, t(38)=2.88, p=.007$, but not on $\tau$, $t(38)=.75, p=.460$, or $\sigma, t(38)=1.32, p<.195$.

\section{Discussion}

The arrow-based Simon effect was obtained on mean RT, and the effect became larger at longer RTs in the Vincentile analyses, replicating findings obtained in previous studies (Pellicano et al., 2009; Proctor et al., 2009; Proctor et al., 2011). As predicted, we obtained the word-based Simon effects on $\mu$ and $\sigma$, and on $\tau$. The arrow-based Simon effect on RT may arise from a shift of the RT distribution and from the tail of the RT distribution in the incompatible condition, compared to that in the compatible condition, suggesting that the arrow-based Simon effect occurred in the fast and slow responses.

Compared to the location-based Simon effect in Experiment 1, the arrow-based Simon effect was larger on $\tau$ and mean RT but not on $\mu$, suggesting that the arrow-based Simon effect had a lesser contribution from rapid automatic activation and a larger contribution from relatively slow responses. Compared to the word-based Simon effect in Experiment 2, the arrow-based Simon effect was larger on $\mu$ and mean RT but not on $\tau$, suggesting that the arrow-based Simon effect had a larger contribution of rapid automatic activation. Moreover, the arrow-based Simon effect on $\sigma$ was not different from the word-based Simon effect and negative location-based Simon effect in absolute value, suggesting that the variances across the RT distribution were not different in these tasks. The results taken together imply that the arrow-based Simon effect is a combination of the location-based and word-based effects. There was no difference from the former on $\mu$ and no difference from the latter on $\tau$. Thus, the ex-Gaussian analysis provides evidence that the arrow-based Simon effect was largest because it had both spatial and symbolic components.

\section{General discussion}

The current study examined whether various Simon effects in different tasks occur on the ex-Gaussian parameters, and whether these effects are different on each parameter. In Experiment 1, we observed a location-based Simon effect on RT, and it decreased from bins 1 to 5 . The ex-Gaussian analysis showed that the location-based Simon effect was on $\mu$ (with a reverse effect on the variability, $\sigma$ ) but not on $\tau$. In Experiments 2 and 3, the word-based and arrow-based Simon effects were obtained on RT, and they increased from bins 1 to 5. The wordbased Simon effect showed approximately similar sized effects on $\mu$ and $\tau$, with the former being smaller than that for the location-based Simon effect and the latter being larger. The arrow-based Simon effect did not differ from that of the location-based Simon effect on $\mu$, but it was larger than it on $\tau$. The reverse relation held for the arrow-based Simon effect in comparison to the wordbased Simon effect. In other words, the largest Simon effect, that for left- and right-pointing arrows, is composed of two components, a normal distribution effect similar to that of spatial-location stimuli and an exponential tail similar to that of location-word stimuli. 
The current findings obtained in the Vincentile analyses are consistent with previous findings that the location-based Simon effect with horizontal S-R arrangement decreases as RTs increase, whereas the word- and arrow-based Simon effects increase (Miles \& Proctor, 2012; Pellicano et al., 2009; Proctor et al., 2009). Moreover, the arrow-based Simon effect occurred for each bin, whereas the word-based Simon effect occurred for the late bins, as shown in Fig. 2 which may be because the arrows activated both visual- and semantic-spatial codes. The visual-spatial codes may provide the basis for the arrow-based Simon effect in the early RT bins, as for the location-based Simon effect. Likewise, the semantic-spatial codes may provide the basis for the arrow-based Simon effect in the late RT bins, as for the word-based Simon effect, as shown in Fig. 2. The conclusion that arrows activate visual-spatial codes as well as semantic-spatial codes differs slightly from that reached by Miles and Proctor (2012) on the basis of correlational analyses for the three types of Simon effects. This difference likely is a consequence of the arrows having a distinct left or right arrowhead in the present study but not in theirs.

The present results are compatible with the dual-route model (De Jong et al., 1994; Hommel, 1997; Kornblum et al., 1990; Ulrich et al., 2015; Zhang et al., 1999; Zorzi \& Umiltà, 1995), which posits that the speed of task-irrelevant spatial location activated automatically, determines the increasing or decreasing delta plots for the various Simon effects. These results in the Vincentile analyses also were compatible with Wascher's theory of separate mechanisms (Pratte et al., 2010; Wascher et al., 2001; Wiegand \& Wascher, 2007; Wiegand \& Wascher, 2005) that proposes a "visuomotor Simon effect" underlying the location-based Simon task and a "cognitive Simon effect" occurring in other forms of the Simon task.

The reverse location-based Simon effect on $\sigma$ showed a larger variability for the compatible than for the incompatible RT distribution, whereas the positive word- or arrow-based Simon effect on $\sigma$ showed a reverse pattern. They are consistent with the simulation in an Appendix of De Jong et al.'s (1994) article and the results of Zhang and Kornblum's (1997) study. Zhang and Kornblum reported a mathematical proof that showed that the decrease or increase of a compatibility effect with the increase of RT is separately determined by whether the variance of the RT distribution for compatible trials is smaller or larger than that for incompatible trials. Therefore, the increasing or decreasing delta plot seems to be linked to the positive or negative Simon effect on $\sigma$, respectively.

Word- and arrow-based Simon effects on $\mu, \sigma$, and $\tau$ were obtained and they were positive, consistent with the finding in the color-word Stroop task (see Balota et al. 2010; Heathcote et al. 1991; Spieler et al. 1996). As shown in Fig. 2, the wordand arrow-based Simon effects on RT arose from both a shift of the RT distribution (a change in $\mu$ ) and the tail of the RT distribution (a change in $\tau$ ) in the incompatible condition, compared to the compatible condition. This pattern suggests that the word- and arrow-based Simon effects occur regardless of whether responding is slow or fast. However, the wordbased Simon effect was smaller than the arrow-based Simon effect on $\mu$ and mean RT but not on $\tau$ and $\sigma$, suggesting that the arrow-based Simon effect on mean RT had a relatively greater contribution from fast responses. The disparity on $\mu$ and mean RT are not explained by Wascher's theory of separate mechanisms (Pratte et al., 2010; Wascher et al., 2001; Wiegand \& Wascher, 2005, 2007), as both the arrow- and word-based Simon effects are classified as cognitive Simon effects in this theory. However, the disparity on $\mu$ and mean RT might be explained by the dual-route model (De Jong et al., 1994; Hommel, 1997; Kornblum et al., 1990; Ulrich et al., 2015; Zhang et al., 1999; Zorzi \& Umiltà, 1995), as the relative speeds of the automatic activation produced by the arrows might be faster than that produced by the location words, given that spatial words are more difficult to interpret compared to arrow stimuli, probably due to computational complexity and attentional requirements underlying the comprehension of the stimulus (Gibson \& Kingstone, 2006).

The location-based Simon effect occurred on $\mu$, whereas a reverse effect was obtained on $\sigma$ and no effect was obtained on $\tau$. They showed that the location-based Simon effect on RT mainly arose from a shift of the RT distribution (a change in $\mu$ ) but not from the tail of the RT distribution (a change in $\tau$ ) in the incompatible condition, compared to that in the compatible condition, as shown in Fig. 1. They suggest that the location-based Simon effect on RT mainly is evident in faster responses but not slower ones. No location-based Simon effect occurring on $\tau$ is in agreement with the view that suggested in prior studies that $\tau$ is associated with response conflict or competition (e.g., Spieler et al. 1996; Steinhauser \& Hübner, 2009; White et al., 2016).

The location-based Simon effect in Experiment 1 and the word-based Simon effect in Experiment 2 were not different on mean RT or on the absolute value of $\sigma$, but the former was larger than the latter on $\mu$ and was smaller than the latter on $\tau$, suggesting that the word-based Simon effect arises more from slow responses, whereas the location-based Simon effect arises mainly from fast responses. This disparity may be because the stimulus location activates visual-spatial codes and the location word activates semantic-spatial codes, and the relative speeds of visual-spatial codes may be faster than the semantic-spatial codes, resulting in the different results on $\mu$, $\sigma$ and $\tau$, which is consistent with the dual-route model (De Jong et al., 1994; Hommel, 1997; Kornblum et al., 1990; Ulrich et al., 2015; Zhang et al., 1999; Zorzi \& Umiltà, 1995). Wascher's theory of separate mechanisms (Pratte et al., 2010; Wascher et al., 2001; Wiegand \& Wascher, $2005,2007)$ also may explain these different results on $\mu, \sigma$, 
and $\tau$, as two different mechanism may be responsible for the location-based and word-based Simon effects, respectively.

Comparing the location-based Simon effect in Experiment 1, the arrow-based Simon effect in Experiment 2 was larger on $\tau$ and mean RT but not on $\mu$ and on the absolute value of $\sigma$, suggesting that the arrow-based Simon effect on mean RT arose from both fast and slow responses, whereas the location-based Simon effect mainly arose from fast responses. This disparity could be explain by the dual-route model (De Jong et al., 1994; Hommel, 1997; Kornblum et al., 1990; Ulrich et al., 2015; Zhang et al., 1999; Zorzi \& Umiltà, 1995), as the stimulus location activate the visual-spatial codes, whereas the arrow activates both the visual-spatial and semantic-spatial codes, which make the spatial properties of arrow activated quickly and its semantic properties develop slowly. Wascher's theory of separate mechanisms (Pratte et al., 2010; Wascher et al., 2001; Wiegand \& Wascher, 2005, 2007) may explain different results on $\sigma$ and $\tau$, while it may not explain indifference on $\mu$ for the location- and arrow-based Simon effects.

These different patterns of results of the Simon effects generated by the three spatial modes on ex-Gaussian parameters suggest that spatial information conveyed by stimulus location, location word and arrow direction has different time courses of activation. However, more research is needed to confirm these findings, given that few prior studies have used the ex-Gaussian approach and the current results were from between-subject or between-experiment comparisons. Moreover, we expect this technique to haves a range of applications to various issues in the field of spatial compatibility effects, given that it provides a clear and precise methodology for studying the pattern of response activation across the RT distribution in compatibility tasks.

In conclusion, the location-based Simon effect occurred on mean RT, $\mu$, and $\sigma$ but not on $\tau$, and the effect was not different from the word-based Simon effect on mean RT but on $\mu, \sigma$, and $\tau$. Moreover, the location-based Simon effect was not different from the arrow-based Simon effect obtained on $\mu$ but on mean RT, $\sigma$, and $\tau$. The arrow-based Simon effect was not different from the word-based Simon effect on $\tau$ and $\sigma$ but on $\mu$ and mean RT. These different results on mean RT and ex-Gaussian parameters provide evidence that spatial information conveyed by physical location and by location word and arrow direction are different in the time courses of activation.

Funding This research was supported by grants from the National Science Foundation of China (31470984).

Compliance with ethical standards Written consent was obtained from all participants prior to participation. The protocol was approved by the institutional review board (IRB) at the Institute of Psychology, Chinese Academy of Sciences.

Conflict of interest The authors have declared that no competing interests exist.

\section{References}

Andrews, S., \& Heathcote, A. (2001). Distinguishing common and taskspecific processes in word identification: A matter of some moment? Journal of Experimental Psychology: Learning, Memory and Cognition, 27, 514-544.

Ansorge, U., \& Wühr, P. (2004). A response-discrimination account of the Simon effect. Journal of Experimental Psychology: Human Perception and Performance, 30, 365-377.

Balota, D. A., \& Spieler, D. H. (1999). Lexicaliry, frequency, and repetition effects: Beyond measures of central tendency. Journal of Experimental Psychology: General, 128, 32-55.

Balota, D. A., Tse, C., Hutchison, K. A., Spieler, D. H., Duchek, J. M., \& Morris, J. C. (2010). Predicting conversion to dementia of the Alzheimer's type in a healthy control sample: The power of errors in Stroop color naming. Psychology and Aging, 25, 208-218.

Davranche, K., \& McMorris, T. (2009). Specific effects of acute moderate exercise on cognitive control. Brain and Cognition, 69, 565-570.

De Houwer, J., Beckers, T., Vandorpe, S., \&Custers, R. (2005). Further evidence for the role of mode-independent short-term associations in spatial Simon effects. Perception \& Psychophysics, 67, 659-666.

De Jong, R., Liang, C.-C., \& Lauber, E. (1994). Conditional and unconditional automaticity: A dual-process model of effects of spatial stimulus-response correspondence. Journal of Experimental Psychology: Human Perception and Performance, 20, 731-750.

Ellinghaus, R., Karlbauer, M., Bausenhart, K.M., \& Ulrich, R. (2017). On the time-course of automatic response activation in the Simon task. Psychological Research, 1-10.

Freitas, A. L., Bahar, M., Yang, S., \& Banai, R. (2007). Contextual adjustments in cognitive control across tasks. Psychological Science, 18, 1040-1043.

Gibson, B. S., \& Kingstone, A. (2006). Visual attention and the semantics of space: Beyond central and peripheral cues. Psychological Science, 17, 622-627.

Heathcote, A., Popiel, S. J., \& Mewhort, D. J. K. (1991). Analysis of response time distributions: An example using the Stroop task. Psychological Bulletin, 109, 340-347.

Hockley, W. E. (1984). Analysis of response time distributions in the study of cognitive processes. Journal of Experimental Psychology: Learning, Memory, and Cognition, 10, 598-615.

Hohle, R. H. (1965). Inferred components of reaction times as functions of foreperiod duration. Journal of Experimental Psychology, 69, 382-386.

Hommel, B. (1997). Interactions between stimulus-stimulus congruence and stimulus-response compatibility. Psychological Research, 59, 248-260.

Hommel, B., Pratt, J., Colzato, L., \& Godijn, R. (2001). Symbolic control of visual attention. Psychological Science, 12, 360-365.

Khalid, S., \& Ansorge, U. (2013). The Simon effect of spatial words in eyemovements: Comparison of vertical and horizontal effects and of eyeand finger responses. Vision Research, 86, 6-14.

Kornblum, S., Hasbroucq, T., \& Osman, A. (1990). Dimensional overlap: Cognitive basis for stimulus-response compatibility-A model and taxonomy. Psychological Review, 97, 253-270.

Kubo-Kawai, N., \& Kawai, N. (2010). Elimination of the enhanced Simon effect for older adults in a three-choice situation: Ageing and the Simon effect in a go/no-go Simon task. Quarterly Journal of Experimental Psychology, 63, 452-464.

Lu, C.-H., \& Proctor, R. W. (1995). The influence of irrelevant location information on performance: A review of the Simon and spatial Stroop effects. Psychonomic Bulletin \& Review, 2, 174-207.

Lu, C.-H., \& Proctor, R. W. (2001). Influence of irrelevant information on human performance: Effects of S-R associations strength and relative timing. Quarterly Journal of Experimental Psychology, 54, 95-136. 
Luo, C., \& Proctor, R. W. (2016). How different location modes influence responses in a Simon-like task. Psychological Research, https://doi. org/10.1007/s00426-22-0809-7

Massidda, D. (2013). retimes: Reaction Time Analysis (R package version 0.1-2). Retrieved from http://CRAN.R-project.org/package= retimes

Miles, J. D., \& Proctor, R. W. (2012). Correlations between spatial compatibility effects: are arrows more like locations or words? Psychological Research, 76, 777-791.

Modern Chinese Frequency Dictionary (1985). Beijing, People's Republic of China: Beijing Language Institute Press.

Moutsopoulou, K., \& Waszak, F. (2012). Across-task priming revisited: Response and task conflicts disentangled using ex-Gaussian distribution analysis. Journal of Experimental Psychology: Human Perception and Performance, 38, 367.

Notebaert, W., De Moor, W., Gevers, W., \& Hartsuiker, R. J. (2007). Newvisuo-spatial associations by training verbo-spatial mappings in the first language. Psychonomic Bulletin \& Review, 14, 11831188.

Pellicano, A., Lugli, L., Baroni, G., \& Nicoletti, R. (2009). The Simon effect with conventional signals: A time-course analysis. Experimental Psychology, 56, 219-227.

Pratte, M. S., Rouder, J. N., Morey, R. D., \& Feng, C. (2010). Exploring the differences in distributional properties between Stroop and Simon effects using delta plots. Attention, Perception, \& Psychophysics, 72, 2013-2025.

Proctor, R. W., Marble, J. G., \& Vu, K.-P. L. (2000). Mixing incompatibly mapped location-relevant trials with location-irrelevant trials: Effects of stimulus mode on the reverse Simon effect. Psychological Research, 64, 11-24.

Proctor, R. W., Miles, J. D., \& Baroni, G. (2011). Reaction time distribution analysis of spatial correspondence effects. Psychonomic Bulletin \& Review, 18, 242-266.

Proctor, R. W., \& Vu, K.-P. L. (2002). Mixing location irrelevant and relevant trials: Influence of stimulus mode on spatial compatibility effects. Memory \& Cognition, 30, 281-294.

Proctor, R. W., Yamaguchi, M., Zhang, Y., \& Vu, K. -P. L. (2009). Influence of visual stimulus mode on transfer of acquired spatial associations. Journal of Experimental Psychology: Learning, Memory, and Cognition, 35, 434-445.

Ratcliff, R. (1978). A theory of memory retrieval. Psychological Review, $85,59-108$.

Ratcliff, R. (1979). Group reaction time distributions and an analysis of distribution statistics. Psychological Bulletin, 86, 446-461.

Servant, M., Montagnini, A., \& Burle, B. (2014). Conflict tasks and the diffusion framework: Insight in model constraints based on psychological laws. Cognitive Psychology, 72, 162-195.

Simon, J. R. (1990). The effects of an irrelevant directional cue on human information processing. In R. W. Proctor \& T. G. Reeve (Eds.), Stimulus-response compatibility: An integrated perspective (pp. 31-86). Amsterdam: North-Holland.

Spieler, D. H., Balota, D.A., \& Faust, M. E. (1996). Stroop performance in healthy younger and older adults and in individuals with dementia of the Alzheimer's type. Journal of Experimental Psychology: Human Perception and Performance, 22, 461-479.

Spieler, D. H., Balota, D. A., \& Faust, M. E. (2000). Levels of selective attention revealed through analyses of response time distributions. Journal of Experimental Psychology: Human Perception and Performance, 26, 506-526.
Steinhauser, M., \& Hübner, R. (2009). Distinguishing response conflict and task conflict in the Stroop task: evidence from ex-Gaussian distribution analysis. Journal of Experimental Psychology: Human Perception and Performance, 35, 1398.

Tipples, J. (2002). Eye gaze is not unique: Automatic orienting in response to uninformative arrows. Psychonomic Bulletin \& Review, 9, 314-318.

Töbel, L., Hübner, R., \& Stürmer, B. (2014). Suppression of irrelevant activation in the horizontal and vertical Simon task differs quantitatively not qualitatively. Acta Psychologica, 152, 47-55.

Tse, C. S., Balota, D. A., Yap, M. J., Duchek, J. M., \& McCabe, D. P. (2010). Effects of healthy aging and early stage dementia of the Alzheimer's type on components of response time distributions in three attention tasks. Neuropsychology, 24, 300-315.

Ulrich, R., Schröter, H., Leuthold, H., \& Birngruber, T. (2015). Automatic and controlled stimulus processing in conflict tasks: superimposed diffusion processes and delta functions. Cognitive Psychology, 78, 148-174.

Umiltà, C., \& Nicoletti, R. (1990). Spatial stimulus-response compatibility. In R. W. Proctor \& T. G. Reeve (Eds.), Stimulus-response compatibility: An integrated perspective (pp. 89-143). Amsterdam: North-Holland.

Vu, K. P. L., Ngo, T. K., Minakata, K., \& Proctor, R. W. (2010). Shared spatial representations for physical locations and location words in bilinguals' primary language. Memory \& Cognition, 38, 713-722.

Wang, H., \& Proctor, R. W. (1996). Stimulus-response compatibility as a function of stimulus code and response modality. Journal of Experimental Psychology: Human Perception and Performance, 22, 1201-1207.

Wascher, E., Schatz, U., Kuder, T., \& Verleger, R. (2001). Validity and boundary conditions of automatic response activation in the Simon task. Journal of Experimental Psychology: Human Perception and Performance, 27, 731-751.

White, D., Risko, E. F., \& Besner, D. (2016). The semantic Stroop effect: An ex-Gaussian analysis. Psychonomic Bulletin \& Review, 23, $1576-1581$

Wiegand, K., \& Wascher, E. (2005). Dynamic aspects of stimulusresponse correspondence: evidence for two mechanisms involved in the Simon effect. Journal of Experimental Psychology: Human Perception and Performance, 31, 453-464.

Wiegand, K., \& Wascher, E. (2007). The Simon effect for vertical S- R relations: Changing the mechanism by randomly varying the $\mathrm{S}-\mathrm{R}$ mapping rule? Psychological Research, 71, 219-233.

Yap, M. J., Balota, D. A., Tse, C., \& Besner, D. (2008). On the additive effects of stimulus quality and word frequency in lexical decision: Evidence for opposing interactive influences revealed by RT distributional analyses. Journal of Experimental Psychology: Learning, Memory, and Cognition, 34, 495-513.

Zhang, J., \& Kornblum, S. (1997). Distributional analysis and De Jong, Liang, and Lauber's (1994) dual-process model of the Simon effect. Journal of Experimental Psychology: Human Perception and Performance, 23, 1543-1551.

Zhang, H., Zhang, J., \& Kornblum, S. (1999). A parallel distributed processing model of stimulus-stimulus and stimulus-response compatibility. Cognitive Psychology, 38, 386-432.

Zorzi, M. \& Umiltà, C. (1995). A computational model of the Simon effect. Psychological Research, 58, 193-205. 